\title{
Prevalencia de caninos retenidos en pacientes que acuden a ICSa
}

\author{
Prevalence of retained canines in patients who attend ICSa
}

\author{
Martha Mendoza Rodríguez ${ }^{a}$, Omar Rodríguez Sierra ${ }^{b}$, Carlo E. Medina Solís $^{c}$, María de \\ Lourdes Márquez Corona ${ }^{d}$, Sandra I. Jiménez Gayosso ${ }^{e}$ y Miriam A. Veras Hernández ${ }^{f}$
}

\begin{abstract}
:
Introduction: Retained teeth are those that are partially or fully developed and lodged inside the jaws. Different studies mention that canines, after the third molars, show a higher prevalence in presenting retention. Justification: The canine is considered an important dental organ for the conformation of the facial mass. The correct position helps the contour and aesthetic appearance of the face, its loss will bring about facial alteration, deficiency in the development of the dental arch and inadequate occlusion. No studies have been found in the state of Hidalgo on the behavior of this dental organ, therefore, the following question is an important reason. What is the prevalence of retained permanent canines and damage to adjacent teeth observed in 250 panoramic and cephalometric radiographs of patients who attend dental care in orthodontic clinics of the Academic Area of Dentistry at the Institute of Health Sciences? Objective: To determine the prevalence of canines retained in the upper jaw and alterations caused to adjacent dental organs. Methodology: Descriptive cross-sectional study in 250 panoramic and cephalic radiographs of patients from 8 to 25 years of age. Results: 250 panoramic and cephalometric measurements were drawn, $153(61.2 \%)$ of the female sex and $97(38.8 \%)$ of the male. The prevalence of retained canines was $62.6 \%(n=313) .15 .6 \%(n=78)$ presented its dilated and curved root, which represents an unfavorable risk for its eruption. 14.8\% $(\mathrm{n}=74)$ presented a mesioangular position and $1 \%(\mathrm{n}=5)$ in a mesiohorizontal position. Damage to adjacent dental organs was $6.6 \%(n=33)$, with the lateral incisor being the most affected with $4 \%(n=20)$. Conclusions: The timely detection of the eruption of the canines will allow a diagnosis and preventive or interceptive treatment, avoiding damage to adjacent teeth, improving the patient's occlusion and facial aesthetics.
\end{abstract}

Keywords:

Canine, retained tooth, rash, prevalence, eruptive disorder, orthodontics

Resumen:

Introducción: Los dientes retenidos son aquellos que se encuentran parcial o totalmente desarrollados y alojados en el interior de los maxilares. Diferentes estudios mencionan, que los caninos, después de los terceros molares, muestran mayor prevalencia en presentar retención. Justificación: Se considera al canino como un órgano dental importante para la conformación del macizo facial. La posición correcta ayuda al contorno y apariencia estética de la cara, su pérdida traerá consigo la alteración facial, deficiencia en el desarrollo del arco dental y una oclusión inadecuada. No se han encontrado estudios en el estado de Hidalgo sobre el comportamiento de este órgano dental, por lo tanto, el siguiente cuestionamiento es razón importante. ¿Cuál es la prevalencia de caninos permanentes retenidos y daños a dientes adyacentes observados en 250 radiografías panorámicas y cefalométricas, de los pacientes que acuden a la atención dental en las clínicas de ortodoncia del Área Académica de Odontología en el Instituto de Ciencias de la Salud? Objetivo: Determinar la prevalencia de caninos retenidos en el maxilar superior y alteraciones causadas a órganos dentales adyacentes. Metodología: Estudio transversal descriptivo en 250 radiografías panorámicas y cefálicas de pacientes de 8 a 25 años de edad. Resultados: Se trazaron 250 panorámicas y cefalométricas, $153(61.2 \%)$ del sexo femenino y 97 (38.8\%) del masculino. La

\footnotetext{
${ }^{a}$ Autor de correspondencia, Universidad Autónoma del Estado de Hidalgo, Instituto de Ciencias de la Salud,

https://orcid.org/0000-0001-7887-7580,Email: martha_mendoza2138@uaeh.edu.mx

${ }^{\mathrm{b}}$ Universidad Autónoma del Estado de Hidalgo, Instituto de Ciencias de la Salud, https://orcid.org/0000-0001-5961-3440,

Email: orodriguezsierra@gmail.com

c Universidad Autónoma del Estado de Hidalgo, Instituto de Ciencias de la Salud, https://orcid.org/0000-0002-1410-9491, Email: cemedinas@yahoo.com

d Universidad Autónoma del Estado de Hidalgo, Instituto de Ciencias de la Salud, https://orcid.org/0000-0001-8313-1169, Email: lulumaco_1@yahoo.com.mx

e Universidad Autónoma del Estado de Hidalgo, Instituto de Ciencias de la Salud, https://orcid.org/0000-0001-7176-9587, Email: sajimga@gmail.com

${ }^{\mathrm{f}}$ Universidad Autónoma del Estado de Hidalgo, Instituto de Ciencias de la Salud, https://orcid.org/0000-0003-3678-5658, Email: miriamalejandra_veras@uaeh.edu.mx
} 
prevalencia de caninos retenidos fue del $62.6 \%(\mathrm{n}=313)$. El 15.6\% (n=78) presentó su raíz dilacerada y curva, lo que representa un riesgo desfavorable para su erupción. El 14.8\% ( $\mathrm{n}=74)$ presentó una posición mesioangular y 1\% ( $\mathrm{n}=5)$ en posición mesiohorizontal. El daño a órganos dentales adyacentes fue del 6.6\% ( $\mathrm{n}=33$ ), siendo el de mayor afección el incisivo lateral con el $4 \%$ ( $\mathrm{n}=20$ ). Conclusiones: La detección oportuna de la erupción de los caninos permitirá realizar un diagnóstico y tratamiento preventivo o interceptivo, evitando daños a dientes adyacentes, mejorando la oclusión del paciente y su estética facial.

\section{Palabras Clave:}

Canino, diente retenido, erupción, prevalencia, trastorno eruptivo, ortodoncia

\section{Introducción}

Los dientes retenidos son aquellos que se encuentran parcial o totalmente desarrollados, quedando alojados en el interior de los huesos maxilares, posterior a la época promedio de erupción ${ }^{1}$.

Un diente impactado es aquel que detiene su trayecto de erupción por un bloqueo o barrera física como: otro diente, tejido óseo, tejidos blandos u otros, que le impiden su migración para alcanzar su posición correcta dentro de las arcadas y en oclusión ${ }^{2}$.

Puede ser detectable clínicamente o bien por estudios de diagnóstico como radiografías, Tomografía Axial Computarizada (TAC), o resonancia magnética ${ }^{3,15}$.

Se considera retención primaria cuando no es posible la identificación clínica del diente por presentar una barrera física, posición inadecuada o desarrollo anormal del mismo, explicando así la interrupción en la erupción del germen dentario que aún no ha aparecido en la cavidad bucal ${ }^{3}$.

Se llama retención secundaria a la detención de la erupción de un diente después de su aparición en la cavidad bucal sin existir una barrera física en el camino eruptivo o posición anormal del diente. Esta anomalía también se conoce como reimpactación, infraoclusión, diente sumergido o hipotrusión, y afecta principalmente a dientes temporales y es rara en dientes permanentes ${ }^{3}$.

Se considera que un diente incluido es cuando permanece dentro del hueso y por tanto, inclusión abarca los conceptos de retención primaria y de impactación ósea ${ }^{3}$. "La importancia de evaluar la impactación, retención e inclusión radica en que el órgano dental mantiene su saco folicular, cuyo epitelio tiene la capacidad de diferenciación con posible progresión a lesiones tumorales (formación de quistes dentígeros, queratoquistes odontogénicos, ameloblastomas, entre otros), además, la estructura dental puede presentar complicaciones como presencia de caries dental y enfermedad pulpar" ${ }^{16}$.

A pesar de que la impactación dentaria puede considerarse como un tipo de erupción ectópica, su principal característica es la ausencia de erupción de uno o más dientes en la cavidad oral. Habitualmente se observan dientes impactados en la dentición permanente; los de mayor frecuencia son los terceros molares inferiores y los caninos superiores, que por su importancia clínica merecen especial atención ${ }^{5}$.

\section{Odontogénesis de los caninos}

La formación del canino superior comienza a los 4 meses \pm 20 días de vida intrauterina continuando su formación posnatalmente durante la dentición decidua. La corona del canino permanente se termina de formar a los 6 o $7 \pm$ años de edad, y la raíz queda formada aproximadamente a los 13.6 \pm años de edad, el periodo de erupción es entre los 11.6 años \pm (5 meses) emergiendo a la cavidad oral una vez que se forma $3 / 4$ de su raíz, no obstante, el canino

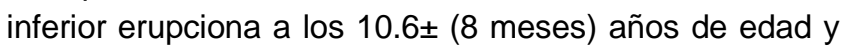
su raíz queda totalmente formada a los 12 años \pm (9 meses) de edad ${ }^{1,6,15}$.

Los caninos son de los últimos dientes en erupcionar dentro de las arcadas dentales, son largos y resistentes debido a su anatomía. La raíz de los caninos superiores es habitualmente, la más larga de todos; es gruesa vestibulingualmente con depresiones de desarrollo por mesial y distal que aseguran el anclaje de este diente en los maxilares ${ }^{1}$.

\section{Etiología de los caninos}

Las etiologías debido a las cuales se presentan inclusiones, retenciones e impactaciones dentarias son de origen desconocido, entre las cuales se pueden clasificar así: locales, generales o genéticas ${ }^{13}$.

Los factores generales 0 genéticos abarcan enfermedades sistémicas, como cuadros febriles, irradiación, síndrome de Gardner, disostosis cleidocraneal, desórdenes genéticos y deficiencias endócrinas ${ }^{7,12}$.

Entre los factores locales están las discrepancias óseodentarias, pérdida temprana o retención prolongada del canino temporal, anquilosis dental, quistes o formaciones neoplásicas, iatrogenia, dientes supernumerarios, dilaceraciones de la raíz, traumatismo, cierre prematuro del ápice, espacio insuficiente en la arcada dental debido a incongruencias esqueléticas (micrognasia), pérdida prematura de dientes deciduos, erupción ectópica, alteraciones bioquímicas en el germen dentario, 
extracciones, retención y/o impedimento para erupcionar ya sea por la obstrucción de hueso o por dientes adyacentes ${ }^{4,11}$.

El canino, por ser el último diente en hacer erupción, es susceptible de presentar problemas de espacio en la arcada, lo que puede dar como resultado su erupción alta más allá del límite mucogingival. Suele condicionar también cierto retraso por obligar al diente a cambiar el trayecto y salir a través de una zona ósea muy condensada (lámina externa) que retarda el proceso eruptivo $^{8,10}$.

\section{Importancia de los caninos}

Los caninos son considerados dientes claves en la oclusión. Estéticamente juegan un papel muy importante, dan armonía facial (influencia en el desarrollo del tercio medio, forma de las arcadas, crecimiento y desarrollo de los arcos, la línea de la sonrisa y surco geniano). Por la longitud y grosor de su raíz, genera una eminencia ósea que se llama eminencia canina, la cual es la responsable de dar soporte al labio superior. Por su posición, influyen directamente en la sonrisa. La ausencia de caninos superiores trae consigo falta de apoyo en los tejidos blandos y labio superior, dando una apariencia de vejez.

La clasificación de los caninos retenidos, según el Dr. Trujillo Fandiño (1990), esquematiza en forma clara y sencilla la retención de estos, así como, también a órganos dentarios retenidos que guardan cierta similitud a los caninos en cuanto a forma y número de raíces, tomando en cuenta sus características tales como:

\section{Posición}

Describe la ubicación de la corona del órgano dentario retenido con relación a los tercios radiculares cervical, medio y apical de los dientes adyacentes, estableciendo 5 $\mathrm{mm}$, para cada tercio radicular.

Posición I: Cuando la corona o la mayor parte de ésta se encuentra a nivel del tercio cervical de la raíz de los dientes adyacentes en los maxilares dentados. $Y$ en espacio comprendido de la cresta alveolar hasta $5 \mathrm{~mm}$ de ésta en el maxilar equivalente al tercio cervical.

Posición II: Cuando la corona o mayor parte de ésta se encuentra a nivel del tercio medio de las raíces de los dientes adyacentes en los maxilares dentados. $Y$ en el espacio comprendido entre 5 y $10 \mathrm{~mm}$ de la cresta alveolar de los maxilares, equivalente al tercio medio.

Posición III: Cuando la corona o la mayor parte de ésta se encuentra a nivel del tercio apical de las raíces de los dientes adyacentes en los maxilares dentados. $Y$ en el espacio existente a partir de $10 \mathrm{~mm}$ de la cresta alveolar de los maxilares. (Fig. 1).

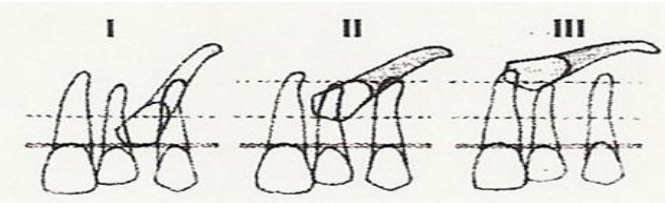

Fig. 1 Trujillo, JJ. Retenciones dentarias en la región anterior. Práctica Odontológica. 1990;11:29-35

\section{Dirección}

Describe la posición de la corona y la inclinación del eje axial, del órgano retenido: vertical, mesioangular, mesiohorizontal, vertical invertido, distoangular, etc. (Fig. 2)

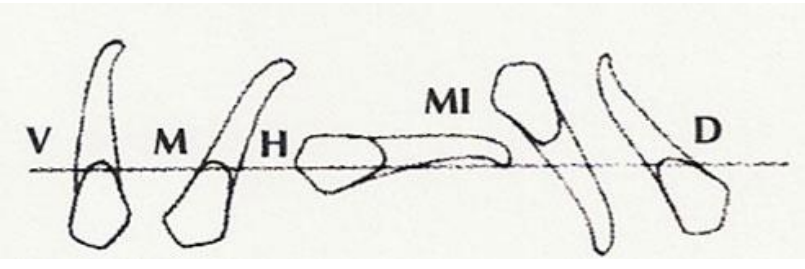

Fig. 2 Trujillo, JJ. Retenciones dentarias en la región anterior. Práctica Odontológica. 1990;11:29-35

\section{Estado radicular}

Describe la morfología radicular: raíz incompleta en su formación, raíz recta, raíz curva, raíz con dilaceración y raíz con hipercementosis. (Fig. 3).

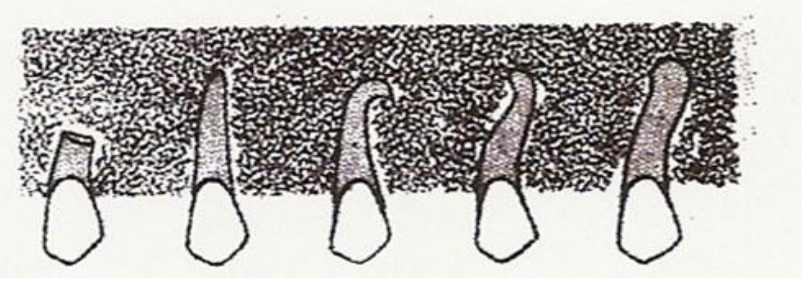

Fig. 3 Trujillo, JJ. Retenciones dentarias en la región anterior. Práctica Odontológica. 1990;11:29-35

\section{Presentación}

Describe la ubicación de la corona según se encuentre dentro de los maxilares: vestibular, palatino o lingual y central.4 (Fig. 4$)^{6}$.

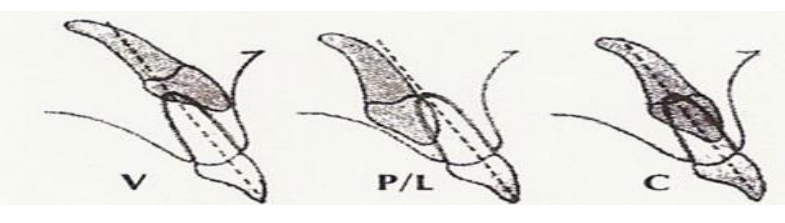

Fig. 4 Trujillo, JJ. Retenciones dentarias en la región anterior. Práctica Odontológica. 1990;11:29-35 
Clasificación de caninos retenidos, según el Dr. Ugalde (2001)

1.- Ubicación de la retención si se encuentra en el maxilar o la mandíbula.

2.- Determinar si la retención es unilateral derecho o izquierdo, o bilateral.

3.- Describir la angulación del canino retenido en relación al plano oclusal: vertical, oblicua u horizontal, con una angulación aproximada de 0 a 30 grados. (Fig. 5).

4.- Estado radicular: en formación, dilacerada y formación completa.

5.- Daño a órganos dentales adyacentes o sin daño9

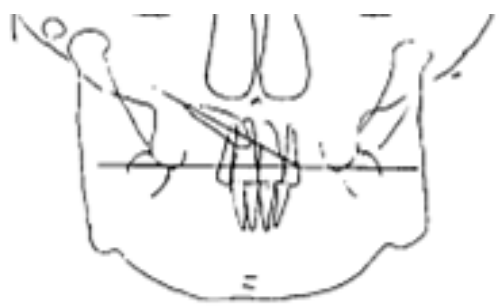

Fig. 5 Ugalde FJ. Clasificación de caninos retenidos y su aplicación clínica. Revista de la ADM. 2001;58(1):21-30.

\section{Metodología:}

Se realizó un estudio observacional descriptivo de 250 radiografías panorámicas digitales y 250 radiografías cefalométricas digitales, seleccionadas por conveniencia, de pacientes de 8 a 25 años de edad, que acudieron a atención dental en las clínicas del Instituto de Ciencias de la Salud, durante el periodo enero-junio 2017, y que llevaron sus radiografías provenientes de un centro radiológico, el cual cuenta con un aparato calibrado. Los criterios de inclusión comprendieron radiografías panorámicas digitales con parámetros adecuados de calidad radiográfica como nitidez, contraste, brillo y distorsión en rangos normales y que permitían observar adecuadamente las estructuras anatómicas objeto de estudio. Como criterios de exclusión se seleccionaron presencia de patologías que podían ser evidenciadas claramente, fracturas de los maxilares, caninos ausentes e imposibilidad de analizar de modo adecuado el diente incluido, retenido o impactado.

Un ortodoncista analizó y revisó las radiografías en un negatoscopio, realizó los trazados en papel de trazado cefalométrico, de acuerdo a la clasificación del Dr. Trujillo y el Dr. Ugalde. Anotando en un formato de registro.

Para el presente estudio se utilizó, la clasificación de caninos retenidos del Dr. Trujillo (1990), que determina la posición, dirección y estado radicular ${ }^{6}$.

También se utilizó la clasificación del Dr. Ugalde y cols. (2001) para la presentación de la corona de los caninos dentro del maxilar por vestibular, palatino o lingual; para determinar la ubicación derecha e izquierda; angulación y daño a los dientes adyacentes ${ }^{9}$.

El análisis estadístico se realizó utilizando el software (SPSS/PC versión 23) la estadística descriptiva fue calculada para las diferentes mediciones.

Resultados: Se trazaron 250 radiografías panorámicas y cefalométricas, $153(61.2 \%)$ fueron del sexo femenino y 97 (38.8\%) masculino. (Gráfica 1) La media de edad fue de $15.87 \pm 4.94$

Gráfica 1. Población por género

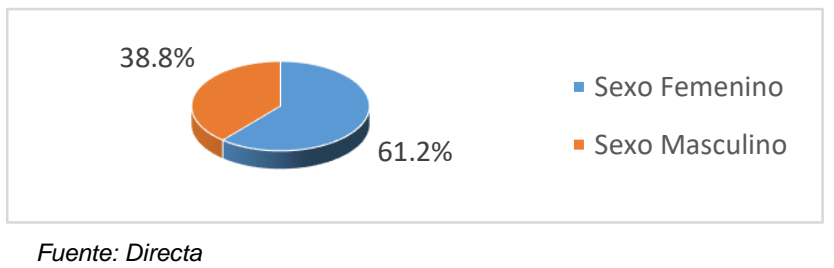

La prevalencia de caninos retenidos fue del $62.6 \%$ (n=313). (Gráfica 2)

Gráfica 2. Prevalencia de caninos retenidos

$\begin{array}{ll}62.6 \% & \\ 62.4 \% & =\text { Retenidos retención }\end{array}$

Fuente: Directa

El $15.6 \%(n=78)$ presentó forma de raíz dilacerada y curva que representa un riesgo desfavorable de erupción, (Gráfica 3)

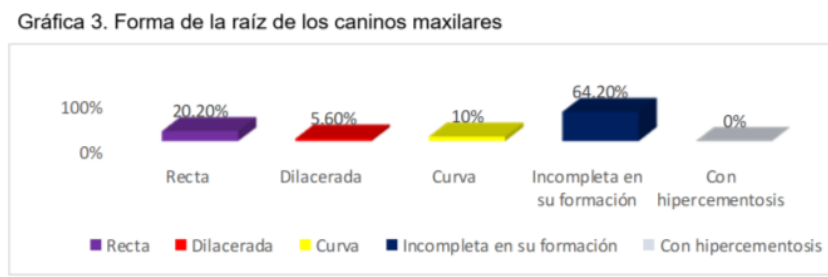

Fuente: Directa

El $14.8 \%(n=74)$ presentó una posición mesioangular y el $1 \%(n=5)$ en una posición mesiohorizontal, ambas representan un mal pronóstico de erupción. (Tabla 1)

Tabla 1. Posición de erupción de la corona e inclinación del eje axial de los caninos maxilares

\begin{tabular}{|c|c|c|c|c|c|}
\hline Dirección & Porcentaje & $\begin{array}{c}\text { Canino } \\
\text { derecho }\end{array}$ & $\begin{array}{c}\text { Canino } \\
\text { izquierdo }\end{array}$ & Prevalencia de caninos \\
\hline Vertical & $420(84 \%)$ & $204(40.8 \%)$ & $216(43.2 \%)$ & $98.8 \%$ & Buen pronóstico \\
\hline Mesioangular & $74(14.8 \%)$ & $43(8.6 \%)$ & $31(6.2 \%)$ & & de erupción \\
\hline Mesiohorizontal & $5(1.0 \%)$ & $3(0.6 \%)$ & $2(0.4 \%)$ & $1.2 \%$ & Mal pronóstico \\
\hline Distoangular & $1(0.2 \%)$ & $0(0 \%)$ & $1(0.2 \%)$ & & de erupción \\
\hline Fuente: Directa & & & & & \\
\hline
\end{tabular}


En cuanto a la ubicación de la corona del canino maxilar, el $1.6 \%(n=8)$ se encuentra por vestibular, el $85 \%(n=425)$ por palatino y el $13.4 \%(n=67)$ por central. (Gráfica 4$)$

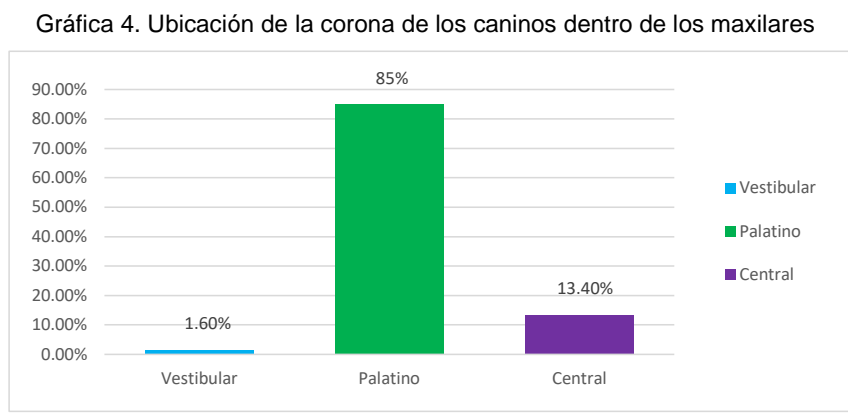

Fuente: Directa

Con respecto a la ubicación de los caninos maxilares en relación a los tercios radiculares de los dientes adyacentes se registraron 374 (74.8\%) en Posición I, 114 (22.8\%) en Posición II y 12 (2.4\%) en Posición III, este último representa un riesgo desfavorable de erupción. (Gráfica 5)

Gráfica 5. Ubicación de la corona de los caninos en relación a los tercios radiculares de los dientes adyacentes

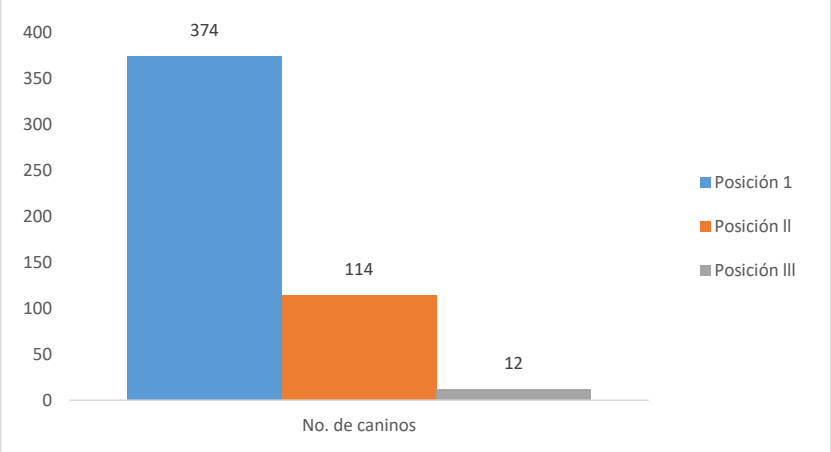

Fuente: Directa

El daño a órganos dentales adyacentes fue del $6.6 \%$ $(n=33)$ siendo el de mayor afección el incisivo lateral con el $4 \%(n=20)$.

\section{Discusiones:}

El apartado de discusiones es complicado, ya que, en los artículos encontrados en estudios similares, la muestra es diferente, así como la clasificación utilizada para medir angulación y posición. La prevalencia de caninos retenidos ha sido reportada por varios autores encontrando diferencias considerables por el tamaño de la muestra, Guere y Silva ${ }^{11}$ reportaron una prevalencia del $5.3 \%(n=44)$; en 828 pacientes; Fernández y Baglini ${ }^{17}$ reportaron una prevalencia de caninos retenidos del $23.28 \%(n=146)$ de un total de 500 pacientes, Castañeda y cols. ${ }^{4}$ reportaron una prevalencia de caninos retenidos del $0.5 \%(n=14)$; en el presente estudio se registró una prevalencia de caninos retenidos del 62.6\% ( $n=313)$, observando un alto porcentaje de retención; no mostrando concordancia con los demás estudios, posiblemente por razones particulares del tamaño de la muestra.

Con respecto a la posición de caninos retenidos dentro de los maxilares, Troya y cols. ${ }^{18}$ en un estudio en 40 pacientes, reportaron que el $60 \%(n=24)$ se encontraron en posición palatina; en el presente estudio se registró una prevalencia de los caninos maxilares del $85 \%(n=425)$ en posición palatina y el $13.4 \%(n=67)$ en posición central, encontrando un porcentaje alto con respecto a la posición palatina comparativamente con Troya y cols.

En relación a la ubicación de la corona de los caninos dentro de los maxilares superiores, Abia $A B .{ }^{19}$ en un estudio realizado en 36 pacientes, reportó que el $22.22 \%$ $(n=16)$ se encontraron por vestibular y el $33.33 \% \quad(n=24)$ por palatino, en este estudio se encontró prevalencia mayor a la encontrada por Abia AB. estimándose el 1.6\% $(n=8)$ por vestibular y el $85 \%(n=425)$ por palatino; diferencia que puede asociarse a características distintas de la muestra estudiadas.

\section{Conclusiones:}

Caninos retenidos es un tema poco abordado a pesar de situarse en el segundo lugar de retenciones dentarias después de los terceros molares. Por lo cual, es muy importante realizar más estudios con la finalidad de dar a conocer las principales características de los caninos retenidos y así obtener un plan de tratamiento adecuado.

La detección oportuna en la erupción de los caninos permitirá realizar un diagnóstico y tratamiento preventivo o interceptivo evitando daños a dientes adyacentes, mejorando la oclusión del paciente y estética facial. Los estudios apoyados en la tecnología como la Tomografía Axial Computarizada (TAC) proporcionan mayor información sobre la posición y ubicación de los dientes en los maxilares.

\section{Referencias}

[1] Aguana, Cohen K, Padrón L. Diagnóstico de caninos retenidos y su importancia en el tratamiento ortodóncico. Revista Latinoamericana de Ortodoncia y Odontopediatría. 2011;1-15.

[2] De Rapaport H, Aichembaum A. Caninos retenidos. Revista Ateneo Argentino de Odontologia (RAAO). 2007; 46(2):10-16.

[3] Escoda C, Berini L. Dientes incluidos. Causas de la inclusión dentaria. Posibilidades terapéuticas ante una inclusión dentaria. Tratado de cirugía bucal. Ergon. 2015;335-337. 
Publicación semestral, Educación y Salud Boletín Científico Instituto de Ciencias de la Salud Universidad Autónoma del Estado de

Hidalgo, Vol. 8, No. 16 (2020) 14-19

[4] Castañeda DA, Briceño CR, Sánchez AE, Rodríguez A, Castro D, Bareientos $S$. Prevalencia de dientes incluidos, retenidos e impactados analizados en radiografías panorámicas de la población de Bogotá, Colombia. Univ Odontol. 2015;34(73):149-157.

[5] Martínez IP, Vera ME, Mora R. Manejo ortodóncico de paciente con desplazamiento de canino asociado a una inusual impactación del incisivo superior. Reporte de caso. Revista Mexicana de Ortodoncia. 2014;2(1):57-66.

[6] Ugalde FJ, González R. Prevalencia de retenciones de caninos en pacientes tratados en la clínica de ortodoncia de UNITEC. Revista de la Asociación Dental Mexicana. 1999;56(2):49-58.

[7] Egido S, Arnau S, Juárez I, Jané E, Marí A, López J. Caninos incluidos, tratamiento odontológico. Revisión de la literatura. Avances en Odontoestomatologia. 2013;29(5):227-238.

[8] Canut Brusola JA. Ortodoncia clínica y terapéutica. Elsevier Masson 2010:60-61.

[9] Ugalde FJ. Clasificación de caninos retenidos y su aplicación clínica. Revista de la Asociación Dental Mexicana. 2001;58(1):21-30.

[10] Cooke J, Wang H. Canine impactions: incidence and management. Int Journal Periodontics Restorative Dent. 2006;26(5):483-491.

[11] Guere I, Silva $R$. Evaluación radiográfica de caninos maxilares impactados, en una muestra de una población mexicana. Revista Latinoamericana de Ortodoncia y Odontopediatría, 2013:34-37.

[12] Khadija S, Zouheir I, Kehoum E. Means of localization of impacted canines: new update. Belge Med Dent. 2010;65(2):87-96.

[13] Martínez A, Diaz A, Fonseca M. Enfoque quirúrgico de canino incluido en el paladar: Reporte de un caso y revisión de la literatura. Revista de la Facultad de Ciencias de la Salud. 2009;6(1):56-61.

[14] Pentón V, Véliz Z, Herrera L. Diente retenido-invertido. Presentación de un caso: modelos de diagnóstico y evaluación. Revista Científica de las Ciencias Médicas en Cienfuegos. 2009;7(6):59-63.

[15] Pérez MA, Pérez P, Fierro C. Alteraciones en la erupción de caninos permanentes. International Journal of Morfhology. 2007;27(1):139143.

[16] Navarro VC. Tratamiento de las inclusiones dentarias. Tratado de Cirugía Oral y Maxilofacial. Arán. 2009;1:19-23.

[17] Fernandez JE, Baglini C. Estudio estadístico retrospectivo de la prevalencia de caninos retenidos. XVII Jornada de Investigación Científica. 2016;333-334.

[18] Troya B, Martinez A, Padilla S, Matos C. Tratamiento quirurgico de caninos retenidos en el Municipio Colón, Cuba. Revista Medica Electrónica. 2016;38(2).

[19] Abia AB. Prevalencia de caninos permanentes incluidos en pacientes de 13 a 17 años del Instituto Nacional de Salud del Niño del 2005 a 2010. [Tesis de licenciatura] Lima (Perú): Universidad Nacional Mayor de San Marcos; 2011 